\title{
Placental Mesenchymal Dysplasia; Current Understanding of the Sonographic, Histologic, and Molecular Findings of a Rare and Challengin Disorder
}

\author{
Ilir Tasha ${ }^{1}$ and Noam Lazebnik ${ }^{2 *}$ \\ ${ }^{1}$ Gynecological Obstetric University Hospital “Koço Gliozheni”, Albania \\ ${ }^{2}$ Department of Obstetrics and Gynecology, University Hospitals Case Medical Center, USA
}

Submission: February 20, 2020; Published: February 25, 2020

*Corresponding author: Noam Lazebnik, Department of Obstetrics and Gynecology, University Hospitals Case Medical Center, USA

\begin{abstract}
Placental mesenchymal dysplasia (PMD) is a rare placental abnormality of yet undetermined etiology. It could be associated with fetal Beckwith-Wiedemann syndrome (BWS), fetal tumors of various organs, fetal growth restriction as well as intrauterine and neonatal death. It is under-diagnosed as many obstetricians and sonologists, are unfamiliar with the clinical entity. This review is aiming at sharing and detailing our experience and that of others of the abnormal sonographic findings, the macroscopic and microscopic pathologic findings, the role of DNA methylation abnormality leading to androgenic uniparental gene expression, resulting in BWS, and the various pregnancy outcomes associated with PMD.
\end{abstract}

Keywords: Placental mesenchymal dysplasia; Sonographic; Histologic; Androgenic uniparental gene expression

Abbreviations: PMD: Placental Mesenchymal Dysplasia; BWS: Beckwith-Wiedemann Syndrome; FGR: Fetal Growth Restriction; SNP: Single Nucleotide Polymorphism

\section{Introduction}

Placental mesenchymal dysplasia (PMD) is a rare placental abnormality/condition of as yet undetermined etiology with an incidence of about 0.02 per 1000 deliveries [1]. PMD was first recognized by Takayama et al. as a distinct pathologic entity of the placenta [2]. Additional reports of unusual placentas with vascular abnormalities were published in the 1980s and 1990s; finally Moscoso et al. [3] recognized the uniqueness of this disorder and described it as a placental vascular anomaly with diffuse stem villous hyperplasia. PMD can be associated with fetal BeckwithWiedemann syndrome (BWS), fetal tumors of various organs, fetal growth restriction as well as intrauterine and neonatal death. It is probably under-diagnosed and under-reported because many healthcare providers, including pathologists and sonologists, are unfamiliar with the clinical entity [4].
We have diagnosed and cared for 4 cases with confirmed PMD in the last 8 years. Three patients elected to terminate the pregnancy while one case continued the pregnancy and experienced premature delivery at 34 weeks. BWS has been confirmed in the newborn. This review is aiming at sharing and detailing our experience and that of others of the abnormal sonographic findings, the macroscopic and microscopic pathologic findings, the role of DNA methylation abnormality leading to androgenic uniparental gene expression, resulting in BWS, and finally the various pregnancy outcomes associated with PMD.

\section{Ultrasound Study Findings}

Although placental PMD related cystic changes may be seen as early as 8 weeks PMD cases are typically detected in the early second trimester once the placental cysts progressively enlarge, 
becoming more numerous and complex as gestation proceeds [5-8] (Figure 1). Low or absent color Doppler signals may be associated with PMD during the first two trimesters (Figure 2). In the third trimester, large vascular areas with turbulent blood flow are observed (either arterial or venous), which are located mainly under and at the level of the chorionic plate. These changes are due to the progressive dilatation of chorionic arteries and veins, which become aneurysmal.

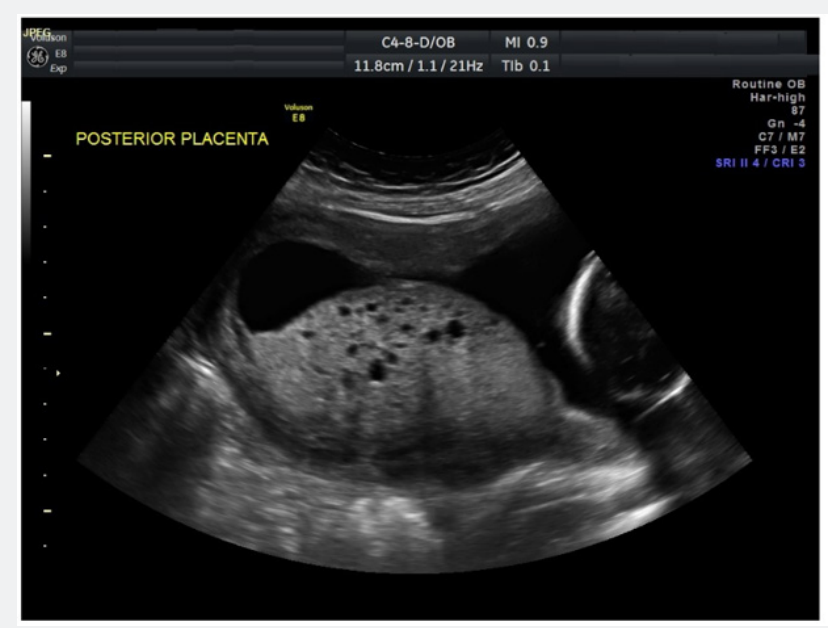

Figure 1: Trans abdominal ultrasound showing a bulky placenta contained a mixed-echogenic mass.

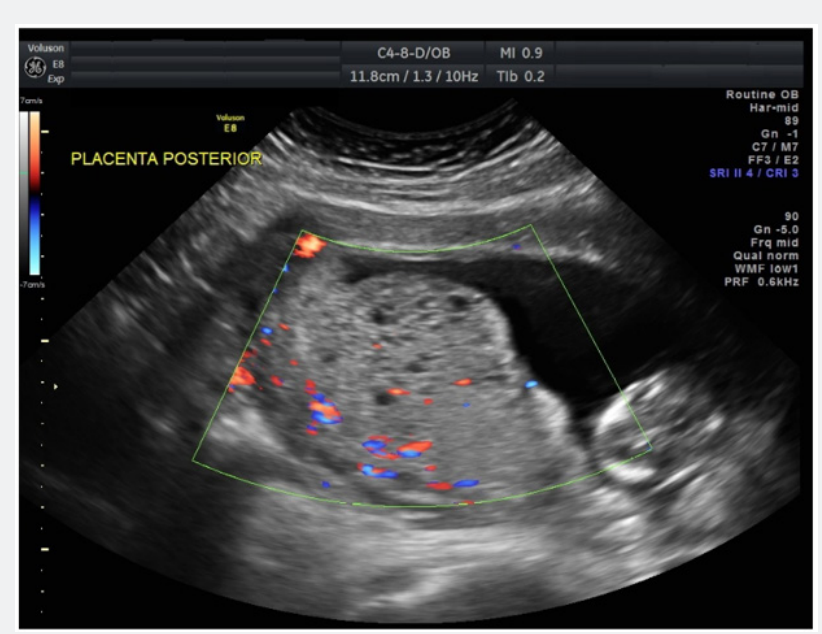

Figure 2: Trans abdominal ultrasound showing color Doppler study of a placenta with multiple cystic echoes without vascular flow.

PMD is characterized by multiple hypoechoic vesicles which are similar to molar changes in the placenta. A characteristic feature of PMD is varicose dilation of chorionic vessels. Gradual reduction in size of PMD's placental vesicular lesions by serial study of placental images has been reported. In contrast, dilated placental vessels on the fetal side became apparent at 38 weeks [9]. Based on similar sonographic findings of enlarged, and thickened placenta with cystic spaces, PMD is most often mistaken for molar pregnancy, chorioangioma, complete mole with coexisting normal fetus, subchorionic hematoma, spontaneous abortion with hydropic changes and partial hydatidiform mole [10]. The utilization of color Doppler might be helpful in differentiate PMD from other cystic placental abnormalities. On color Doppler, high velocity and low resistance flow is seen in the molar mass and large feeding vessel or increased vascularity is seen in the mass of chorioangioma, while no blood flow is seen within the mass in subchorionic hematoma, and spontaneous abortion with hydropic changes. Differentiation of PMD from a twin pregnancy with a complete mole and coexistent fetus is difficult. On first trimester ultrasound study, documentation of two placental masses, a lesion that constitute the entire thickness of the placenta, and lack of blood flow signals suggest the diagnosis of complete mole with coexistent fetus [11]. While there is no clear evidence to suggest that Doppler interrogation of placental and umbilical vessels is 
helpful in recognizing and confirming cases of PMD, it does play a crucial role in cases complicated by fetal growth restriction (FGR).

It is of utmost importance to realize that sonographic findings indicative of PMD warrant careful evaluation of the fetus and placenta to rule out the presence of polyhydramnios, placentomegaly, macrosomia, macroglossia, omphalocele, visceromegaly, hemi hypertrophy, as well as fetal hepatic and lung mesenchymal tumor $[12,13]$.

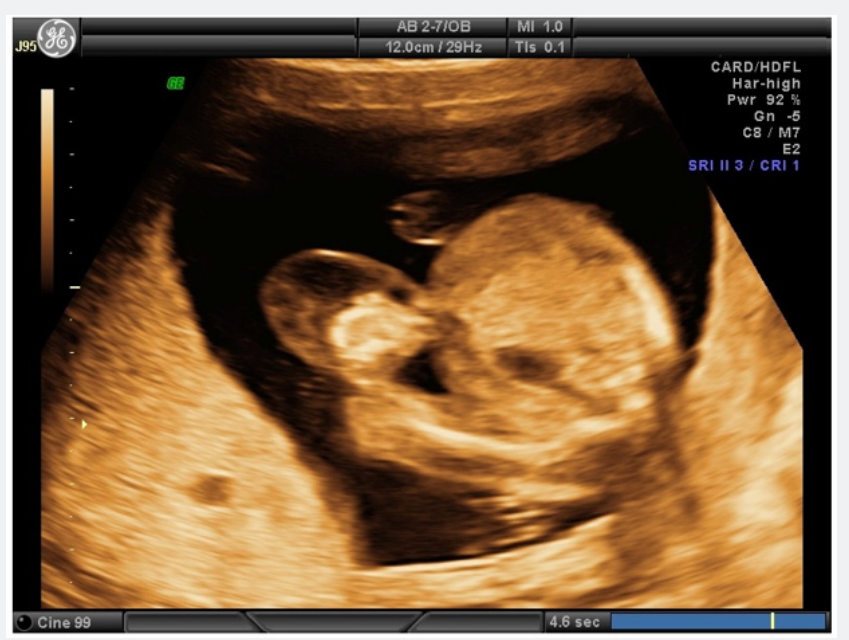

Figure 3: Fetal omphalocele is seen at 13 weeks gestation in a PMD case.

The characteristic sonographic placental abnormalities (Figure 1) and fetal omphalocele (Figure 3) are both amenable to first trimester sonographic finding and are routinely observed in early gestation with close to $100 \%$ accuracy. Associated findings of macrosomia, macroglossia, and visceromegaly, when present, are detected later in gestation. Thus, Imaging in later gestation is warranted as it may reveal additional abnormalities that support a BWS diagnosis. An unusual sonographic finding of interest is a case reported of dilatation of the umbilical vein with elevated maternal serum alpha-fetoprotein on prenatal testing that was later confirmed to be PMD on pathological evaluation of the placenta [14].

\section{Differential Diagnosis}

The differential diagnosis of PMD is broad and includes partial molar pregnancy, complete mole with co-existing normal fetus, hemangioma, subchorionic hematoma, confined placental mosaicism and hydropic placental changes following extended period of time of spontaneous undiagnosed miscarriage. The observation of intra placental 'cysts', together with the presence of an embryo or fetus, frequently leads obstetricians to misdiagnose this condition as a partial mole. While the appearance of cysts in molar pregnancy is caused by the presence of edematous villous stroma (hydropic villi), the cysts in PMD are dilated (aneurismal) vessels. The hypoechoic areas also correspond to cistern formation in dysplastic stem villi present in placental parenchyma.

In order to help with the sonographic suspicion of PMD, Kuwata $\mathrm{T}$ et al. [15] have suggested that in the second and third trimesters color Doppler study be used to show blood flow within the placental cysts, leading to what the authors refer to 'stained-glass' appearance, a finding which might be helpful in differentiating PMD from other forms of placental cysts. The placenta of a complete mole with coexisting normal fetus and partial molar pregnancy appears heterogeneous, with partially solid and cystic areas. On ultrasound study, a chorioangioma is a focal lesion and is hypoechoic compared to the rest of the placenta and is typically located on the fetal surface of the placenta.

Acase of a normal viable fetus with concomitant chorioangioma and PMD, (frequently the two are seen together) has been reported [16]. The case was first identified by prenatal ultrasonography, but the prenatal diagnosis only included chorioangioma. PMD was then confirmed during postnatal evaluation of the placenta, which included gross and microscopic examination of the placenta. Another case of co-occurrence of chorioangioma and PMD has been reported to be associated with maternal pre-eclampsia and fetal growth restriction [17].

Surti U et al. have reported of an unusual trizygotic pregnancy that resulted in live-born twins suggesting diverse etiology of PMD cases [18]. The placenta of one twin had placental mesenchymal dysplasia (PMD), which resulted from a chimeric fusion of an androgenetic zygote and a normal biparental zygote. One placenta was noted to be diffusely cystic and enlarged. The macroscopic and microscopic findings were consistent with the diagnosis of PMD; however, genetic findings confirmed confined placental chimerism involving a normal biparental $46, \mathrm{XY}$ male conceptus 


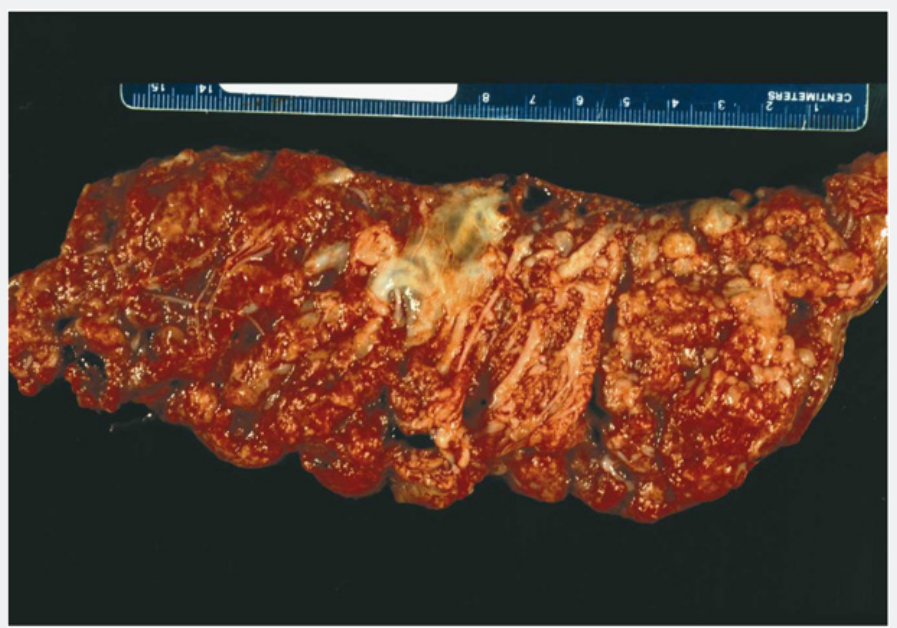

Figure 4: Gross specimen of a placental mesenchymal dysplasia weighing $1315 \mathrm{~g}$.

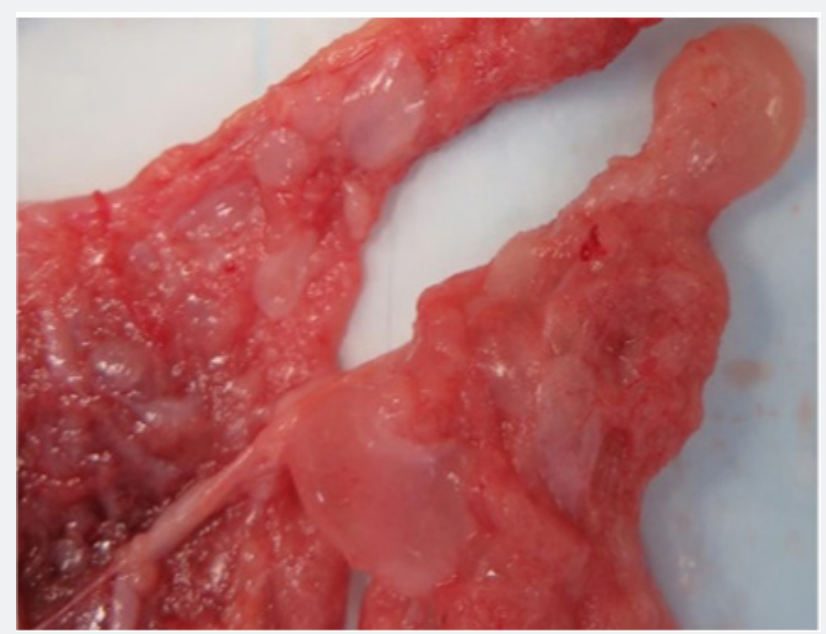

Figure 5: Gross photograph of placenta, demonstrating multiple thin-walled cysts.

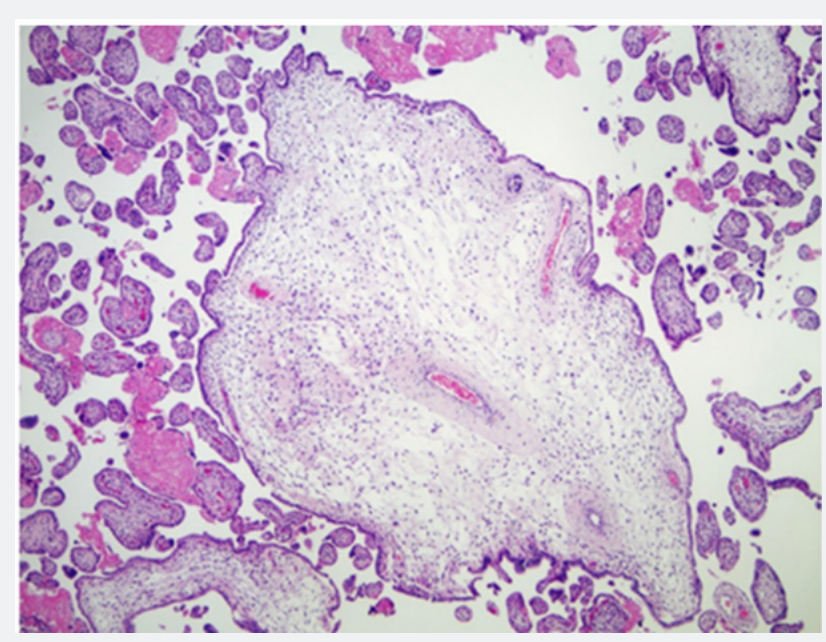

Figure 6: Abnormal appearing stem villi with stromal overgrowth and thick-walled fetal vessels and relatively normal appearing surrounding uninvolved villi. Hematoxylin and eosin stain. Original magnification $\times 10$. 


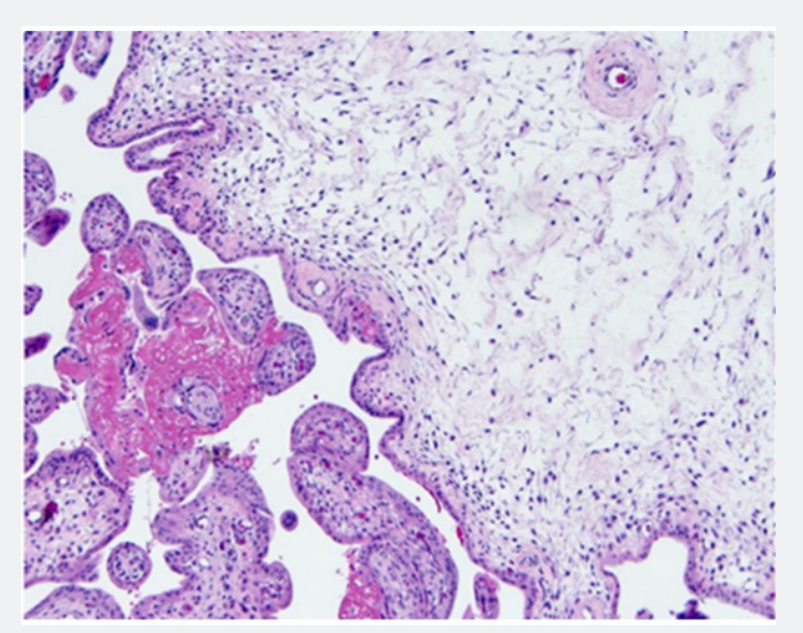

Figure 7: Stem villous with stromal overgrowth without trophoblast hyperplasia and relatively normal appearing surrounding uninvolved villi. Hematoxylin and eosin stain. Original magnification $\times 10$.

The diagnosis of PMD is only affirmed after evaluation of placental pathology. Grossly, it is characterized by placentomegaly (Figure 4), dilated or even aneurysmal chorionic vessels and enlarged hydropic or cystic villi (Figure 5). Microscopic findings include mesenchymal hyperplasia and edema of stem-cell villi, which contain thick-walled vessels (Figure 6). A characteristic feature is the absence of trophoblastic hyperplasia (Figure 7), which is a hallmark of gestational trophoblastic disease $[7,10,21]$.

When PMD is associated with BWS excessive cord length, hyper-coiling, and/or varix formation is particularly common; however, placentomegaly and insertional, umbilical arterial, and cord-coiling abnormalities also occur in PMD cases without BWS [22]. Faye-Petersen OM, et al. [23] have reported on the main histolopathologic findings of PMD which include the following placental tissue abnormalities:

a) Chorionic plate and stem villous vascular dilatation and foci of vascular mural attenuation, myxomatous change, with or without luminal compromise attributable to thrombi of varying age.

b) Clusters of abnormally vascularized stem villi.

c) Large to distal branch stem villous stromal hypocellularity and cistern formation.

d) Hypervascularized, distal stem, and intermediate villi with concentration of abnormal, thickly muscularized to thrombosed vessels subjacent to the trophoblast (vs the centralized location of vessels seen in normal villi).

e) Hydropic and degenerated villi scattered among intervening more normal-appearing villi.

\section{Molecular Genetic Abnormalities}

Genomic imprinting is an epigenetic phenomenon that leads to parent-specific differential expression of a subset of mammalian genes. Multiple imprinted genes have been identified in humans and mice, and most imprinted genes often form clusters, or imprinting domains. The expression of imprinted genes within these domains is regulated by imprinting control regions (ICRs) [24,25]. ICRs are identical to differentially methylated regions (DMRs), which are characterized by DNA methylation on one of the two parental alleles, maternally methylated DMRs or paternally methylated DMRs. Gametic DMRs acquire DNA methylation during gametogenesis, and the methylation is maintained from zygote to somatic cells during all developmental stages. Methylations of somatic DMRs are established during early embryogenesis after fertilization under the control of nearby ICRs. As most imprinted genes have an important role in the growth and development of embryos, placental formation, and metabolism, aberrant expression of imprinted genes due to epigenetic or genetic abnormalities has been implicated in the pathogenesis of human disorders such as congenital anomalies and tumors24, 25. Epigenetic abnormality leading to aberrant expression of imprinted genes would result in hypomethylation or hypermethylation at ICRs.

Representative of imprinting disorders and their corresponding imprinted loci include the BWS at 11p15.5, Prader-Willi/Angelman syndromes at 15q11-q13, pseudopara hypothyroidism type $1 \mathrm{~b}$ at 20q13.3, Silver-Russell syndrome (SRS) at 11p15.5 and chromosome 7, and transient neonatal diabetes mellitus type 1 at 6q24. 
BWS is associated with abnormal regulation of multiple genes transcription in two imprinted domains on chromosome 11p15.5 (also known as the BWS critical region). The BWS critical region includes two domains: Imprinting center 1 (IC1) regulates the expression of IGF2 and H19 in domain 1 and imprinting center 2 (IC2) regulates the expression of CDKN1C, KCNQ10T1, and KCNQ1 in domain 2. Other genetic alteration include genomic variants involving chromosome $11 \mathrm{p} 15.5$ are cytogenetically visible duplications, inversions or translocations, or copy number variants including microduplications or microdeletions of $11 \mathrm{p} 15.5$

Androgenetic-biparental mosaicism (ABM), in which a subset of cells in the placenta are diploid but harbor only paternal chromosomes, has been the most consistent molecular alteration observed in PMD [26-29]. The androgenetic cells in ABM have pan-genomic paternal uniparental disomy (paternal alleles and imprinting at all loci), and detection of allelic imbalances consistent with $\mathrm{ABM}$ is considered confirmatory evidence for the diagnosis of PMD.
Studies have revealed that the anomalous, but diploid villi of PMD have androgenetic stromal cells [23]. In addition to ABM, some cases of PMD may result from a mosaic distribution of placental cells with segmental forms of uniparental disomy, possibly restricted to the BWS locus on chromosome 11p15.5 [30]. Immunohistochemical staining for the CDKN1C gene product, p57KIP2, provides an indirect, light microscopic assessment for global allelic imbalance in ABM or segmental loss of maternal 11p15.5 imprinting. Because the CDKN1C gene on chromosome $11 \mathrm{p} 15.5$, encodes a cyclin dependent kinase inhibitor, p57KIP2, which is expressed from the maternally acquired chromosome, but methylated (silenced) on the paternally inherited genome [31]. The encoded protein is a tight-binding, strong inhibitor of several G1 cyclin/Cdk complexes and a negative regulator of cell proliferation. Diseases associated with CDKN1C mutations include BWS, fetal growth retardation, metaphyseal dysplasia, adrenal hypoplasia congenita, and genital anomalies.

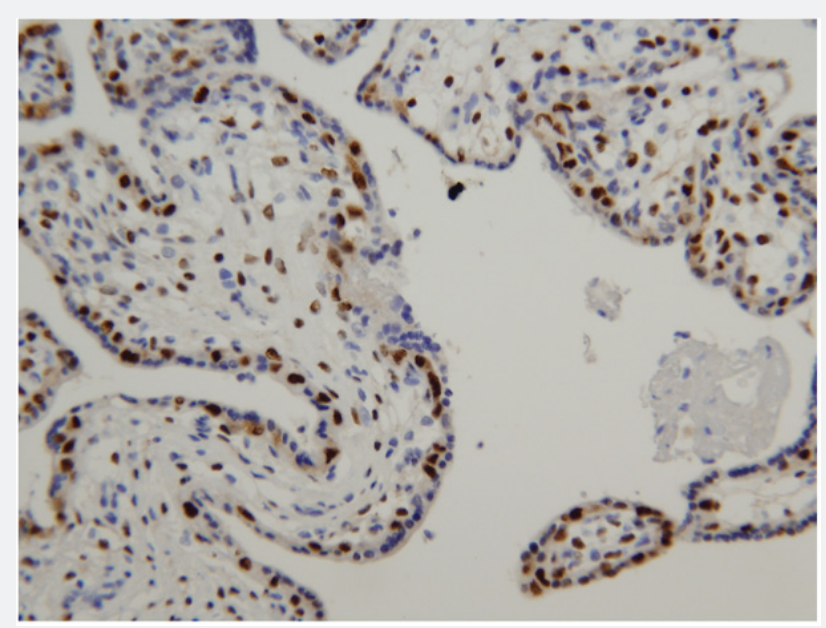

Figure 8a: Positive staining for p57KIP2 in trophoblast and stromal cells in normal pregnancy. Original magnification $\times 10$.

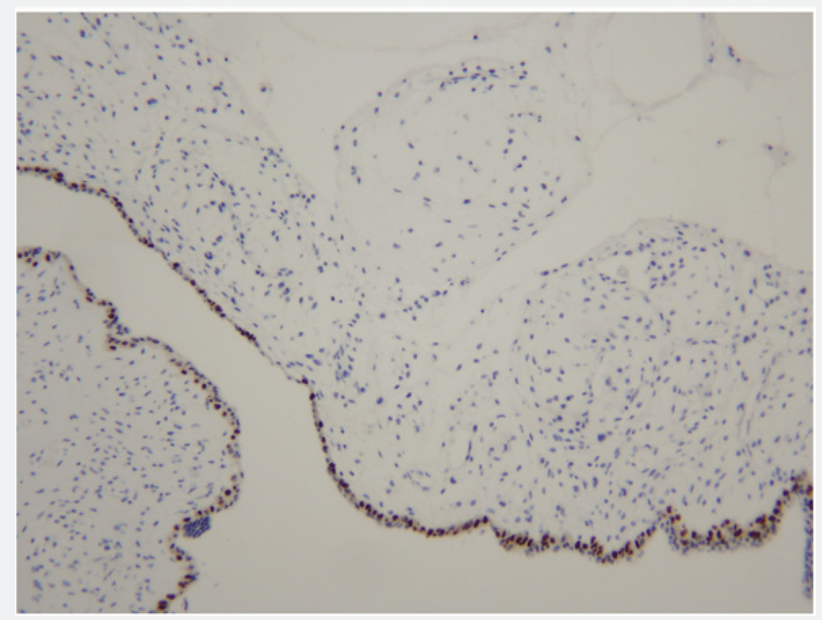

Figure 8b: Normal trophoblast staining for p57KIP2 but absent stromal staining in placental mesenchymal dysplasia. Original magnification $\times 10$. 
The trophoblast is composed of perivillous trophoblast (cytotrophoblast and syncytiotrophoblast), and extravillous intermediate trophoblast. P57 is always positive in theintermediate trophoblast and always negative in the syncytiotrophoblast. The typical case of PMD shows absence of p57kip2 nuclear immunostaining in portions of the stroma, mainly composed of mesenchymal cells that display loss of p57 expression in PMD of abnormally vascularized stem villi, but positive linear staining (presence of the product of the maternally derived active allele) in the overlying villous surface trophoblast. On the other hand nondysmorphic villi in the section exhibit nuclear staining within the stroma and trophoblast (normal staining pattern). These findings are consistent with androgenetic allelic imbalance in villi with the morphology of PMD, but not within the normal appearing villi (Figure 8a\&b).

It is important to emphasize that because the distribution of androgenetic cells and associated PMD histopathology is rarely uniformly present, villous sampling might have limitations in some cases for molecular genetic or immune-histochemical studies. An optional way of overcoming such limitation is studying the placental tissue for the presence or absence of p57KIP2 a product of the CDKN1C gene.

In an appropriately sampled case, with convincing PMD histomorphology, but a negative or inconclusive immune-histochemical staining pattern for the p57KIP2 gene product followup molecular genetic studies should be strongly considered [23]. Fluorescence in situ hybridization studies using polymorphic allele-specific probes, provide an alternative approach, but are not as widely practiced as molecular tests that rely on DNA extracts from fresh, frozen, or fixed tissue [32,33].

A recent report have suggested that vascular endothelial growth factor (VEGF)-D may be associated with the development of PMD a disorder characterized by cell proliferation/differentiation $[34,35]$. This gene located on chromosome Xp22.31 is regulating cell proliferation and/or differentiation, The expression of five cell-proliferation/differentiation-associated genes, namely, Wnt3a, Wnt5a, $\beta$-catenin, VEGF-D and Dickkopf-1 (DKK-1) were examined. Expressions of Wnt3a, Wnt5a and $\beta$-catenin were decreased, whereas those of VEGF-D and DKK-1 were increased. These abnormal expressions might suggest a relationship between these genes and PMD pathogenesis/pathophysiology.

\section{PMD and Fetal Aneuploidy}

The majority of studies published to date have reported low association between PMD and chromosome abnormality. There has been only a single reported case of triploidy associated with PMD [36]. Other chromosomally abnormal cases reported included trisomy $13(47, \mathrm{XX}, \mathrm{t}(1: 13)(\mathrm{q} 32 ; \mathrm{q} 32)+13)$ [37], and a
$228 \mathrm{~kb}$ deletion at $13 \mathrm{q} 12.11$ involving the gap junction beta- 6 (GJB6) gene detected by single nucleotide polymorphism (SNP) microarray analysis has been reported [38]. In a review study by Cohen MC et al. [36] Klinefelter syndrome $(47, \mathrm{XXY})$ has been diagnosed with PMB.

Robinson WP reported of a case of PMD associated with a male fetus showing overgrowth with an enlarged heart, marked fetal ascites and intrauterine fetal death at 34 weeks, but no other BWS manifestations. Mosaicism for an unbalanced translocation leading to deletion of the maternal copy of the BWS region on 11p15.5 and partial duplication of $17 \mathrm{q}$ was observed in the placenta, but not in the fetal samples. The authors concluded that while the placental findings of PMD can be caused by an unbalanced dosage of genes in 11 p15.5 alone, fetal growth parameters appear to depend on the underlying mechanism and likely also the level and distribution of abnormal cells [30].

\section{Pregnancy Complications and Outcome}

Pregnancy outcomes has been reported to range from healthy, uncomplicated pregnancies, to adverse maternal and/or neonatal complications $[36,39]$. The majority of studies reporting outcomes among cases with confirmed PMD report poor fetal outcome and multiple maternal complications. However, normal fetal outcomes have been reported mainly as random case reports [40]. In a recent study the maternal, fetal and newborns complications of 22 cases with confirmed PMD were reported [41]. The maternal complications included preeclampsia/gestational hypertension, and premature delivery. Fetal complications included sever fetal growth restriction in half of the cases. In the same study fetal death was reported to be high (about 18\%), and one third of the newborns died within two months of extra uterine life. Similar outcomes were reported by Nayeri UA et al. [19].

In an attempt to explain the high incidence of fetal growth restriction and demise Pham et al. proposed that IUGR and IUFD may occur because of obstructive vascular thrombosis and decreased maternal-fetal gas exchange owing to a reduction in the normal amount of chorionic villi [41].

A rare form of maternal complication has been recently reported. A 20-year-old primigravida presented at 16 weeks with heavy clotty vaginal bleeding and subsequent miscarriage. Post miscarriage, ultrasound study revealed enlarged and multicystic ovaries but no ascites similar to those seen in ovarian hyperstimulation syndrome (OHSS). Follow up ultrasound study several weeks later noted normal ovaries and no ascites. The author suggested that high levels of VEGF known to accompany PMD, despite low levels of serum hCG, resulted in OHSS unlike a case of triploidy that usually presents with high serum hCG level $[42,43]$. 
Multiple forms of fetal anomalies have been reported with PMD regardless of whether BWS was diagnosed or not. These included CHARGE syndrome, fetal pleuro-pulmonary blastoma and fetal skeletal dysplasia [40]. The long-term sequelae of infants delivered in the setting of PMD are unknown. The impact of angiogenic and anti-angiogenic factors has not been fully explored among cases with PMD [40,44]. Poor pregnancy outcome resulting in inutero death of a normally growing fetus have also been reported to be associated with dilated periumbilical chorionic vessels and PMD [45]. Another reported case associated with ruptured vessels and death of a normal size fetus with no congenital anomalies has been reported. In this case as the pregnancy advanced, tangled congested vessels were identified within the placental parenchyma and were most prominent in the subchorionic plate region near the fetal surface at the placental cord insertion. These cases might suggest that ruptured placental choroid vessels are potential treat and can lead to fetal demise $[46,47]$. Severe fetal anemia and thrombocytopenia caused by placental microangiopathic hemolysis in fetuses with normal growth and early childhood development patterns were reported to be associated with PMD [48]. A case of a phenotypically normal neonate with anemia and thrombocytopenia associated with PMD has been reported. Histologic examination of the placenta showed findings consistent with PMD, as well as chorangioma. The co-existence of PMD with fetal hepatic, pulmonary, pancreatic and renal mesenchymal tumors has been reported, suggesting a common patho-genetic origin for these anomalies due to synchronous abnormal development of the mesoderm [36,49-54].

\section{PMD and Multiple Gestations}

Rare cases of PMD including the previously mentioned report by Surti U et al. [18] have been reported to affect the placenta of one fetus in the setup of dichorionic diamniotic twins as well as monochorionic diamniotic twins. PMD associated with fetal growth restriction in one placenta of a dichorionic diamniotic twin pregnancy have been reported. Ultrasound and magnetic resonance imaging showed one placenta of the growth retarded fetus was bulky and had multiple cysts, while the other fetus placenta appeared normal. Macroscopically, chorionic vessels on the placental surface of the smaller neonate were prominently enlarged [55]. Pathological histologic findings demonstrated swelling stem villi with enlarged vessels and increased interstitial cells without trophoblast proliferation. Immunostaining for p57kip2 was negative in interstitial cells and cytotrophoblasts of the swelling stem villi. This suggested that PMD occurred in one placenta of the dichorionic diamniotic twin, leading to early-onset growth retarded fetus [56-58].

In the case involving monochorionic diamniotic twins pathological examination confirmed a monochorionic diamniotic twin placenta with normal findings in the placental part of twin A. In the part of twin $\mathrm{B}$, the chorionic vessels were much dilated. In the stem villi, the stroma was severely hydropic with the formation of central cisterns. Starting from the stem villi and extending into the intermediary and terminal villi, there was also a diffuse capillary proliferation (chorangiomatosis). No trophoblastic hyperplasia was seen. In the stem villi, the trophoblastic cells were p57 positive, whereas the stromal fibroblasts were p57 negative, thus confirming discordancy for placental mesenchymal dysplasia in a monochorionic placenta [59].

\section{Conclusion}

PMD is associated with adverse pregnancy outcome regardless of the presence or absence sonographic detectable fetal abnormality. Women with PMD are at markedly increased risk of intrauterine fetal death and premature delivery [60]. The diagnosis of PMD should be considered with specific sonographic findings, including enlarged, cystic placenta with dilated chorionic vessels, more so when abnormal levels of biochemical analytes, identified as part of aneuploidy screening, especially elevated maternal serum alpha feto-protein, a finding which further support the diagnosis. Karyotype should be obtained to exclude partial molar pregnancy, as this is the most common misdiagnosis of PMD cases. A detailed anatomical survey should be performed to rule out fetal anomalies, especially abnormalities consistent with BWS as well as liver and lung abnormalities. Fetal growth assessment is mandatory in PMD cases regardless of normal fetal organs [61].

Patients should be counseled regarding potential complications such as fetal growth restriction, fetal death, premature delivery and maternal pre-eclampsia. Serial growth scans starting in the second trimester and continue until delivered should be strongly considered. Early admission to the hospital and intensive monitoring of fetal wellbeing status should be considered, although whether this policy improves outcome has not been validated by carefully designed quality study to date. Finally the placenta should be sent for pathological evaluation after delivery for confirmation of PMD.

\section{Acknowledgement}

The authors would like to acknowledge the clinical expertise and help of Raymond Redline, M.D. Professor of Pathology, University Hospital of Cleveland, who interpreted and provided the pathology slides included in the manuscript.

\section{References}

1. Zeng, X, Chen, MF, Bureau, YA, Brown, R (2012) Placental mesenchymal dysplasia and an estimation of the population incidence. Acta Obstet Gynecol Scand 91(6): 754-757.

2. Takayama M, Soma H, Yaguchi S, Funayama H, Fujiwara K, et al. (1986) Abnormally large placenta associated with Beckwith- Wiedemann syndrome. Gynecol Obstet Invest 22(3): 165-168. 
3. Moscoso G, Jauniaux E, Hustin J (1991) Placental vascular anomaly with diffuse mesenchymal stem villous hyperplasia. A new clinicopathological entity? Pathol Res Pract 187(2-3): 324-328.

4. Guenot C, Kingdom J, Rham DM, Osterheld M, Keating S, et al. (2019) Placental mesenchymal dysplasia: An underdiagnosed placental pathology with various clinical outcomes. Eur J Obstet Gynecol Reprod Biol 234: 155-164.

5. Lee G, Chi JG, Cha KS (1991) An unusual venous anomaly of the placenta. Am J Clin Pathol 95(1): 48-51.

6. Heazell AE, Sahasrabudhe N, Grossmith AK, Martindale EA, Bhatia K, et al. (2009) A case of intrauterine growth restriction in association with placental mesenchymal dysplasia with abnormal placental lymphatic development. Placenta 30(7): 654-657.

7. Umazume T, Kataoka S, Kamamuta K, Tanuma F, Sumie A, et al. (2011) Placental mesenchymal dysplasia, a case of intrauterine sudden death of fetus with rupture of cricoid periumbilical chorionic vessels. Diagn Pathol 6: 38.

8. Jauniaux E, Nicolaides KH, Hustin J (1997) Perinatal features associated with placental mesenchymal dysplasia. Placenta 18(8): 701-706.

9. Ohira S, Ookubo N, Tanaka K, Takatsu A, Kobara H, et al. (2013) Placental mesenchymal dysplasia: chronological observation of placental images during gestation and review of the literature. Gynecol Obstet Invest 75(4): 217-223.

10. Parveen Z, Tongson Ignacio J, Fraser C, Killeen J, Thompson K, et al. (2007) Placental mesenchymal dysplasia. Arch Pathol Lab Med 131(1): 131-137.

11. Giorgione V, Cavoretto P, Cormio G, Valsecchi L, Vimercati A, et al. (2017) Prenatal diagnosis of twin pregnancies with complete hydatidiform mole and coexistent normal fetus: A series of 13 cases. Gynecol Obstet Invest 82(4): 404-409.

12. Shieh HF, Estroff JA, Barnewolt CE, Zurakowski D, Tan WH, et al. (2019) Penatal imaging throughout gestation in Beckwith-Wiedemann syndrome. Prenat Diagn 39(9): 792-795.

13. Harris K, Carreon CK, Vohra N, Williamson A, Dolgin S, et al. (2013) Placental mesenchymal dysplasia with hepatic mesenchymal hamartoma: a case report and literature review. Fetal Pediatr Pathol $32(6): 448-453$

14. Mulch AD, Stallings SP, Salafia CM (2006) Elevated maternal serum alpha fetoprotein, umbilical vein varix, and mesenchymal dysplasia: are they related? Prenat Diagn 26(8): 659-661.

15. Kuwat T, Takahashi H, Matsubara S (2014) 'Stained-glass' sign for placental mesenchymal dysplasia. Ultrasound Obstet Gynecol 43(3) 355-355.

16. Qichang W, Wenbo W, Liangkai Z, Hui K, Xiaoqin H, et al. (2013) Pregnancy with concomitant chorioangioma and placental mesenchymal dysplasia: a rare placental abnormality. Case Rep Obstet Gynecol 23: 591-593.

17. Rosefort A, Cordier AG, Kaddioui S, Beaumont B, Baergen R, et al. (2013) Co-occurrence of multifocal chorioangiomatosis and mesenchymal dysplasia in preeclampsia. Pediratr Dev Pathol 16(3): 206-209.

18. Surti U, Hill LM, Dunn J, Prosen T, Hoffner L, et al. (2005) Twin pregnancy with a chimeric androgenetic and biparental placenta in one twin displaying placental mesenchymal dysplasia phenotype. Prenat Diagn 25(11): 1048-1056.

19. Nayeri UA, West AB, Grossetta NHK, Copel JA, Sfakianaki AK, et al. (2013) Systematic review of sonographic findings of placental mesenchymal dysplasia and subsequent pregnancy outcome. Ultrasound Obstet Gynecol 41(4): 366-374.
20. Elias KM, Berkowitz RS, Horowitz NS (2019) State-of-the-Art Workup and Initial Management of Newly Diagnosed Molar Pregnancy and Postmolar Gestational Trophoblastic Neoplasia. J Natl Compr Canc Netw 17(11): 1396-1401.

21. Paradinas FJ, Sebire NJ, Fisher RA, Rees HC, Foskett M, et al. (2001) Pseudopartial moles: placental stem vessel hydrops and the association with Beckwith-Wiedemann syndrome and complete moles. Histopathology 39(5): 447-454.

22. Baergen RN (2011) Manual of pathology of the human placenta. In: ( $2^{\text {nd }}$ edn), Springer, New York, USA, p. 220-420.

23. Faye-Petersen OM, Kapur RP (2013) Placental Mesenchymal Dysplasia. Surg Pathol Clin 6(1): 127-151.

24. Abramowitz LK, Bartolomei MS (2012) Genomic imprinting: recognition and marking of imprinted loci. Curr Opin Genet 22(2): 7278.

25. Tomizawa S, Sasaki H (2012) Genomic imprinting and its relevance to congenital disease, infertility, molar pregnancy and induced pluripotent stem cell. J Hum Genet 57(2): 84-91.

26. Sebire NJ, Fisher RA (2005) Partially molar pregnancies that are not partial moles: additional possibilities and implications. Pediatr Dev Pathol 8(6): 732-733.

27. Gibson BR, Muir-Padilla J, ChampeauxA, Suarez ES (2004) Mesenchymal dysplasia of the placenta. Placenta 25(7): 671-672.

28. Kaiser-Rogers KA, McFadden DE, Livasy CA, Dansereau J, Jiang R, et al. (2006) Androgenetic/biparental mosaicism causes placental mesenchymal dysplasia. J Med Genet 43(2): 187-192.

29. McConnell TG, Murphy KM, Hafez M, Vang R, Ronnett BM, et al. (2009) Diagnosis and subclassification of hydatidiform moles using p57 immunohistochemistry and molecular genotyping: validation and prospective analysis in routine and consultation practice settings with development of an algorithmic approach. Am J Surg Pathol 33(6): 805811.

30. Robinson WP, Slee J, Smith N, Murch A, Watson SK, et al. (2007) Placental mesenchymal dysplasia associated with fetal overgrowth and mosaic deletion of the maternal copy of 11p15.5. Am J Med Genet 143(15): 1752-1759.

31. Matsuoka S, Thompson JS, Edwards MC, Bartletta JM, Grundy P, et al. (1996) Imprinting of the gene encoding a human cyclin dependent kinase inhibitor, p57KIP2, on chromosome 11p15. Proc Natl Acad Sci USA 93(7): 3026-3030.

32. Chiang S, Fazlollahi L, Nguyen A, Betensky RA, Roberts DJ, et al. (2011) Diagnosis of hydatidiform moles by polymorphic deletion probe fluorescence in situ hybridization. J Mol Diagn 13(4): 406-415.

33. Bourque DK, Penaherrera MS, Yuen RK, Van Allen MI, McFadden DE, et al. (2011) The utility of quantitative methylation assays at imprinted genes for the diagnosis of fetal and placental disorders. Clin Genet 79(2): 169-175.

34. Takahashi H, Matsubara S, Kuwata T, Saruyama M, Usui R, et al. (2014) Changes in expression of vascular endothelial growth factor D-related genes in placental mesenchymal dysplasia. J Obstet Gynaecol Res 40(4): 1145-1149

35. Kotani T, Sumigama S, Tsuda H, Mano Y, Yamamoto E, et al. (2012) A case report of placental mesenchymal dysplasia with an increased VEGF-D expression. Placenta 33(10): 888-891.

36. Cohen MC, Roper EC, Sebire NJ, Stanek J (2005) Anumba DOC. Placental mesenchymal dysplasia associated with fetal aneuploidy. Prenat Diagn 25(3): 187-192. 
37. Müngen E, Dundar O, Muhcu M, Haholu A, Tunca Y, et al. (2008) Placental mesenchymal dysplasia associated with trisomy 13: sonographic findings. J Clin Ultrasound 36(7): 454-456.

38. Johnson SL, Walters-Sen LC, Stanek JW (2018) Placental Pathology in Placental Mesenchymal Dysplasia with 13q12.11 Deletion and a 25Week Gestation Female Infant. Am J Case Rep 19: 369-373.

39. Pal S, Bose K, Ch Mondal P, Chakrabarti S, Sikder M, et al. (2017) Placental Mesenchymal Dysplasia With Normal Fetus: A Rare Case Report. Iran J Pathol 12(3): 307-310.

40. Guenot C, Kingdom J, De Rham M, Osterheld M, Keating S, et al. (2019) Placental mesenchymal dysplasia: An underdiagnosed placental pathology with various clinical outcomes. Eur J Obstet Gynecol Reprod Biol 234: 155-164.

41. Pham T, Steele J, Stayboldt C, Chan L, Benirschke K, et al. (2006) Placental mesenchymal dysplasia is associated with high rates of intrauterine growth restriction and fetal demise: a report of 11 new cases and a review of the literature. Am J Clin Pathol 126(1): 67-68.

42. Davoudian P (2015) Placental mesenchymal dysplasia associated with spontaneous ovarian hyper stimulation syndrome. BMJ Case Rep.

43. Ludwig M, Gembruch U, Bauer O, Diedrich K (1998) Ovarian hyper stimulation syndrome (OHSS) in a spontaneous pregnancy with fetal and placental triploidy: information about the general pathophysiology of OHSS. Hum Reprod 13(8): 2082-2087.

44. Herraiz I, Simón E, Toldos Ó, Rodríguez Y, Gómez-Arriaga PI, et al. (2017) Angiogenesis-related biomarkers (sFlt-1/PlGF) in placental mesenchymal dysplasia. J Matern Fetal Neonatal Med 30(8): 958-959.

45. Yamada A, Sakaida N, Okamura A, Yamada T, Ota T, et al. (2014) Placental mesenchymal dysplasia, a case of intrauterine sudden death. Kobe J Med Sci 60(1): E1-4.

46. Akbarzadeh-Jahromi M, Sari Aslani F, Parvari S (2013) Placental mesenchymal dysplasia complicated by hydrops fetalis and fetal death: a case report. Arch Iran Med 16(9): 551-554.

47. Chen CP, Hsu CY, Su YN, Wang TY, Chern SR, et al. (2013) Placental mesenchymal dysplasia associated with antepartum hemorrhage, subchorionic hematoma, and intrauterine growth restriction. Taiwan J Obstet Gynecol 52(1): 154-156.

48. Sengers FB, Lijnschoten VG, Sluijs-Bens VDJP, Porath MM, Dijkman KP, et al. (2010) Haematological abnormalities in premature babies due to placental mesenchymal dysplasia. Ned Tijdschr Geneeskd 154: A1040.

49. Carta M, Maresi E, Giuffr`e M, Catalano G, Piro E, et al. (2005) Congenital hepatic mesenchymal hamartoma associated with mesenchymal stem villous hyperplasia of the placenta: case report. J Pediatr Surg 40(5) E37-E39.
50. Chan YF, Sampson A (2003) Placental mesenchymal dysplasia: a report of four cases with differentiation from partial hydatidiform mole. Aust N Z J Obstet Gynaecol 43(6): 475-479.

51. Francis B, Hallam L, Kecskes Z, Ellwood D, Croaker D, et al. (2007) Placental mesenchymal dysplasia associated with hepatic mesenchymal hamartoma in the newborn. Pediatr Dev Pathol 10(1): 50-54.

52. Kapur RP, Cole B, Zhang M, Lin J, Fligner CL, et al. (2013) Placental mesenchymal dysplasia and fetal renal-hepatic-pancreatic dysplasia: androgenetic-biparental mosaicism and pathogenesis of an autosomal recessive disorder. Pediatr Dev Pathol 16(3): 191-200.

53. Mack-Detlefsen B, Boemers TM, Groneck P, Bald R (2011) Multiple hepatic mesenchymal hamartomas in a premature associated with placental mesenchymal dysplasia. J Pediatr Surg 46(8): e23-25.

54. Tortoledo M, Galindo A, Ibarrola C (2010) Placental mesenchymal dysplasia associated with hepatic and pulmonary hamartoma. Fetal Pediatr Pathol 29(4): 261-270.

55. Koga H, Makimura M, Tanaka H, Sumioki H (2014) Placental mesenchymal dysplasia and fetal hematologic disorder. J Pediatr Hematol Oncol 36(6): e389-391.

56. Shiro M, Kojima F, Ota N, Minami S, Ino K, et al. (2018) Placental mesenchymal dysplasia with severe fetal growth restriction in one placenta of a dichorionic diamniotic twin pregnancy. J Obstet Gynaecol Res 44(5): 951-954.

57. Jitsumori S, Shiro M, Kojima F, Ota N, Minami S, et al. (2018) Placental mesenchymal dysplasia with severe fetal growth restriction in one placenta of a dichorionic diamniotic twin pregnancy. J Obstet Gynaecol Res 44(5): 951-954.

58. Robertson M, Geerts LT, de Jong G, Wainwright H (2007) Mesenchymal dysplasia in a monochorionic diamniotic twin pregnancy with review of the differential diagnosis of cystic changes in the placenta. J Ultrasound Med 26(5): 689-693.

59. Gheysen W, Strybol D, Moerman P, Steylemans A, Corveleyn A, et al. (2018) Discordance for placental mesenchymal dysplasia in a monochorionic diamniotic twin pregnancy: A case report. Clin Case Rep 6(8): 1557-1560.

60. Ishikawa S, Morikawa M, Yamada T, Akaishi R, Kaneuchi M, et al. (2015) Prospective risk of stillbirth in women with placental mesenchymal dysplasia. J Obstet Gynaecol Res 41(10): 1562-1568.

61. Arigita M, Illa M, Nadal A, Badenas C, Soler A, et al. (2010) Chorionic villus sampling in the prenatal diagnosis of placental mesenchymal dysplasia. Ultrasound Obstet Gynecol 36(5): 644-645. 


\section{Your next submission with Juniper Publishers} will reach you the below assets

- Quality Editorial service

- Swift Peer Review

- Reprints availability

- E-prints Service

- Manuscript Podcast for convenient understanding

- Global attainment for your research

- Manuscript accessibility in different formats

( Pdf, E-pub, Full Tsext, Audio)

- Unceasing customer service

Track the below URL for one-step submission https://juniperpublishers.com/online-submission.php 